\title{
Prediction of B-cell epitopes using evolutionary information and propensity scales
}

\author{
Scott Yi-Heng Lin ${ }^{1}$, Cheng-Wei Cheng ${ }^{2}$, Emily Chia-Yu Su${ }^{3 *}$ \\ From The Eleventh Asia Pacific Bioinformatics Conference (APBC 2013) \\ Vancouver, Canada. 21-24 January 2013
}

\begin{abstract}
Background: Development of computational tools that can accurately predict presence and location of B-cell epitopes on pathogenic proteins has a valuable application to the field of vaccinology. Because of the highly variable yet enigmatic nature of B-cell epitopes, their prediction presents a great challenge to computational immunologists.
\end{abstract}

Methods: We propose a method, BEEPro (B--cell epitope prediction by evolutionary information and propensity scales), which adapts a linear averaging scheme on 16 properties using a support vector machine model to predict both linear and conformational B-cell epitopes. These 16 properties include position specific scoring matrix (PSSM), an amino acid ratio scale, and a set of 14 physicochemical scales obtained via a feature selection process. Finally, a three-way data split procedure is used during the validation process to prevent over-estimation of prediction performance and avoid bias in our experiment results.

Results: In our experiment, first we use a non-redundant linear B-cell epitope dataset curated by Sollner et al. for feature selection and parameter optimization. Evaluated by a three-way data split procedure, BEEPro achieves significant improvement with the area under the receiver operating curve (AUC) $=0.9987$, accuracy $=99.29 \%$, mathew's correlation coefficient $(M C C)=0.9281$, sensitivity $=0.9604$, specificity $=0.9946$, positive predictive value $(\mathrm{PPV})=0.9042$ for the Sollner dataset. In addition, the same parameters are used to evaluate performance on other independent linear B-cell epitope test datasets, BEEPro attains an AUC which ranges from 0.9874 to 0.9950 and an accuracy which ranges from $93.73 \%$ to $97.31 \%$. Moreover, five-fold cross-validation on one benchmark conformational B-cell epitope dataset yields an accuracy of $92.14 \%$ and AUC of 0.9066 .

Conclusions: Compared with other current models, our method achieves a significant improvement with respect to AUC, accuracy, MCC, sensitivity, specificity, and PPV. Thus, we have shown that an appropriate combination of evolutionary information and propensity scales with a support vector machine model can significantly enhance the prediction performance of both linear and conformational B-cell epitopes.

\section{Background} Introduction

The idea of using peptide-based vaccines to replace live or attenuated whole-pathogen vaccines has been an emerging field, as peptide-based vaccines can offer greater safety, potency, and elegance in drug design and delivery [1]. The development of these peptide-based vaccines requires first

\footnotetext{
* Correspondence: emilysu@tmu.edu.tw

${ }^{3}$ Graduate Institute of Biomedical Informatics, Taipei Medical University, Taipei, Taiwan

Full list of author information is available at the end of the article
}

of all the identification of highly immunogenic regions within a given pathogen protein. These immunogenic regions, or more particularly the B-cell epitopes, are responsible for eliciting humoral immune response by inducing production of neutralizing antibodies.

Two types of B-cell epitopes have been defined: linear (continuous) and conformational (discontinuous). While the majority ( $90 \%$ ) of the B-cell epitopes is conformational, the difficulties in the design of such conformational epitopes have led to an emphasis on classification of linear B-cell epitopes [1].
C Biomed Central

(c) 2013 Lin et al.; licensee BioMed Central Ltd. This is an open access article distributed under the terms of the Creative Commons Attribution License (http://creativecommons.org/licenses/by/2.0), which permits unrestricted use, distribution, and reproduction in any medium, provided the original work is properly cited. 
As development of vaccines is critical in our protection against infectious diseases, effective screening methods to identify immunogenic epitopes from the pathogenic proteome will be necessary. Classical methods such as phage display system have successfully yielded peptides that have proceeded to clinical trials, yet these experimental techniques are labour-intensive and may not reflect in vivo binding conditions or the biological ability to stimulate antibody production $[2,3]$. The shortcomings of current experimental methods call for the development of new computational models that can more effectively predict the presence and location of immunogenic (protective) epitopes given a pathogenic protein sequence.

\section{Previous works}

Sollner et al. have studied the use of conservation in posttranslational modification and sequence variability to predict protective linear B-cell epitopes, i.e., linear B-cell epitopes associated with biological activity. Their results showed that focusing on conserved region and regions lacking post-translational modification sites may be beneficial [2]. Training the same dataset using a Naïve Bayes classifier, El-Manzalawy et al. also showed that position specific scoring matrix (PSSM) offered the best performance when compared with selected physicochemical scales and dipeptide composition representation [3]. Together, these two studies demonstrated that protective linear epitopes may have sequence conservations that explain their functional role, and that focusing on protective linear epitopes may improve the performance of current prediction models for linear B-cell epitopes [1].

In addition, Blythe and Flower have previously shown that simple propensity scale-based methods are only marginally better than random prediction [4]. Thus, researches since then have suggested the use of a combination of more than one propensity scale with a machine-learning algorithm to improve the prediction performance [5-7].

\section{Challenges and our contributions}

As the current lack of success in B-cell prediction could be explained by inadequate or incomplete selection of appropriate propensity scales to reflect the complex patterns of B-cell epitopes, our study attempts to first reconfirm the belief that combinational approach outperforms single propensity scale approach. Then, twenty properties, including amino acid ratio scale, PSSM, and 18 physicochemical scales selected from AAIndex, are used to construct a hybrid propensity scale model by training a support vector machine (SVM) classifier on a protective linear B-cell epitope dataset [2]. Parameter optimization and feature selection are then applied to yield an optimal set of propensity scales with the best performance. We propose the final optimized model, BEEPro (B-cell Epitope prediction using Evolutionary information and Propensity scales). Six B-cell epitope datasets are used to evaluate the performance of BEEPro, with one of the datasets consisting of conformational B-cell epitopes.

\section{Methods}

\section{Datasets}

In this study, we applied seven datasets used in previous studies to allow unbiased validation of our method and to compare the performance of our model with others. Table 1 summarizes these datasets, which are detailed below and available in the supplementary material [Additional files 1, 2, 3, 4, 5, 6, 7].

\section{Sollner dataset}

This dataset was curated by Sollner et al. and contains 57 non-redundant pathogen proteins extracted from IEDB database [2]. Each antigen is annotated with a number of linear B-cell epitopes that are classified as "leading to biological activity." This is the first dataset that closely approximates protective linear B-cell epitopes [3]. The dataset is comprised of 2,317 residues labeled as part of an epitope (5.04\%) and 43,690 nonepitope residues (94.96\%). To evaluate the performance of B-cell epitope prediction, this non-redundant dataset is used for feature selection and parameter optimization based on a three-way data split procedure.

\section{AntiJen \#1 and \#2 datasets}

These two datasets were extracted from the AntiJen database [8]. AntiJen\# 1 is provided by Larsen et al. and contains 124 protein sequences (5,529 epitope residues, $8.34 \%$; 60,800 non-epitope residues, $91.66 \%$ ) [7]. AntiJen\#2 is provided by Wang et al. and contains 171 protein sequences with 691 non-overlapping epitopes $(11,249$ epitope residues, $12.92 \%$; 75,805 non-epitope residues, $87.08 \%$ ) [9].

\section{HIV dataset}

This dataset was curated from the HIV Molecular Immunology Database of the Los Alamos National

Table 1 Summary of B-cell epitope datasets used in this study.

\begin{tabular}{|c|c|c|c|c|c|c|c|}
\hline Dataset & Sollner & AntiJen\#1 & AntiJen\#2 & HIV & Pellequer & $\mathrm{PC}$ & Benchmark \\
\hline Number of proteins & 57 & 124 & 171 & 10 & 14 & 12 & 52 \\
\hline Epitope residue & 2,317 & 5,529 & 11,249 & 1,018 & 858 & 1,852 & 858 \\
\hline Non-epitope residue & 43,690 & 60,800 & 75,805 & 1,693 & 1,695 & 3,509 & 9,527 \\
\hline Number of residues & 46,007 & 66,329 & 87,054 & 2,711 & 2,553 & 5,361 & 10,385 \\
\hline Epitope density & $5.04 \%$ & $8.34 \%$ & $12.92 \%$ & $37.55 \%$ & $33.61 \%$ & $34.55 \%$ & $8.26 \%$ \\
\hline
\end{tabular}


Laboratory, http://www.hiv.lanl.gov/[10]. The electronic copy of this dataset is provided by Larsen et al. and contains 10 HIV proteins (1,018 epitope residues, 37.55\%; 1,693 non-epitope residues, 62.45\%) [7].

\section{Pellequer dataset}

This dataset was first presented by Pellequer et al. [11], and the electronic version of this dataset was recreated by Lund et al. [7]. This dataset contains 14 proteins and 83 epitopes (858 epitope residues, 33.61\%; 1,695 nonepitope residues, $66.39 \%$ ).

\section{$P C$ dataset}

This dataset was curated by Wang et al. and contains 12 protein sequences with 98 non-overlapping epitopes ( 1,852 epitope residues, $34.55 \%$; 3,509 non-epitope residues, 65.45\%) [9]. The epitopes in this dataset were experimentally identified with peptide scan methodology [9].

\section{Benchmark dataset}

The original benchmark dataset contains 161 protein chains from 144 antigen-antibody complex structures [12]. Ansari and Raghava applied CD-HIT at $40 \%$ cutoff value to yield a non-redundant dataset of 52 antigen chains (858 epitope residues, $8.26 \%$; 9,527 non-epitope residues, $91.74 \%$ ) $[13,14]$. Epitope residues in this dataset are defined as antigen residues where at least one of the atoms is distanced within $4 \AA$ from any antibody atom based on PDB structures [13].

\section{Calculation of amino acid ratio propensity scale}

The amino acid ratio (AAR) propensity scale for each of the 20 types of amino acid $\alpha_{i}$ is computed according to the following equation, where $f\left(\alpha_{i}^{+}\right)$and $f\left(\alpha_{i}^{-}\right)$represent the occurrence frequencies of amino acid $\alpha_{i}$ in epitope and non-epitope peptide sequences, respectively.

$$
p\left(\alpha_{i}\right)=\frac{f\left(\alpha_{i}^{+}\right) / \sum_{i} f\left(\alpha_{i}^{+}\right)}{f\left(\alpha_{i}^{-}\right) / \sum_{i} f\left(\alpha_{i}^{-}\right)}
$$

Previous studies have used a similar equation to compute an amino acid dimer (or amino acid pairs, AAP) propensity scale $[9,15]$. In those studies, logarithm was taken of the AAP ratios before the normalization step. We do not, however, find significant changes in performance of our hybrid propensity scale model when logarithm is used. Considering that $f\left(\alpha_{i}^{+}\right)$could become zero after data-split (for example, the Benchmark dataset contains only one cysteine epitope residue for the entire dataset), the logarithm step is neglected in this study.

To avoid dominance of any individual $\rho\left(\alpha_{i}\right)$ values, the following equation is used to normalize the values to the range of $[-1,1]$.

$$
\rho\left(\alpha_{i}\right)=2\left(\frac{p\left(\alpha_{i}\right)-\min \left\{p\left(\alpha_{i}\right)\right\}}{\max \left\{p\left(\alpha_{i}\right)\right\}-\min \left\{p\left(\alpha_{i}\right)\right\}}\right)-1
$$

To avoid bias in our results, for all the methods below which involve amino acid ratio propensity scale, the scale values are re-calculated for each fold of cross-validation and three-way data split using only the data of training set.

\section{Generation of position specific scoring matrix}

PSSM is used to reflect the evolutionary information of a peptide. Blast-2.2.26+ package are downloaded from $\mathrm{ftp} / / / \mathrm{ftp} . n \mathrm{ncbi}$.nih.gov/blast/, and the psiblast program of this package is used to generate multiple sequence alignment against non-redundant $(n r)$ protein database, which is downloaded from ftp://ftp.ncbi.nih.gov/blast/ $\mathrm{db} /$. The $n r$ database uploaded on July 9, 2012 is used for this study. PSSM is generated using the setting: evalue $=0.001$, number of iterations $=3$.

\section{Single propensity scale method}

Each peptide of running window size $w$ for a residue at position $i$ is represented by a vector of size $w:\left[x_{i-(w-1) / 2} \ldots\right.$, $\left.x_{i}, \ldots, x_{i+(w-1) / 2}\right]$, where $x_{i}$ is the propensity scale value at residue position $i . w$ in this study ranges from 5 to 29 in steps of 2. For the residues at the $\mathrm{N}$ - or C-terminus of the peptide sequence, appropriate number of zeros is appended to the head or the tail of the vector, respectively, to make up a vector of the right size. In addition to the amino acid ratio propensity scale, four more representative physicochemical scales are also used for comparison: Parker's hydrophilicity [16], Karplus' flexibility [17], Grantham's polarity [18], and Janin's accessible area [19]. These scales have been used by previous studies for B-cell epitope prediction $[3,5,7,13]$.

In the case of PSSM, each peptide of running window size $w$ is represented by a vector of size $[20 \times w]$ because the amino acid at each residue position is represented by an evolutionary information vector of $20 \log$-likelihood values. As in the case of single propensity scale method, zeros are appended at either the head or the tail of the vector for residues at the $\mathrm{N}$ - or the $\mathrm{C}$-terminus of the peptide, respectively, to account for the asymmetry at the two ends of peptide.

\section{Hybrid propensity scale method}

Other than the amino acid ratio propensity scale and PSSM, additional 18 physicochemical scales selected from AAIndex [20] are considered for incorporation into a hybrid propensity scale model. These 18 scales include antigenicity [21], hydrophilicity [16,22], hydrophobicity [23], accessible surface area [19], flexibility $[17,24]$, interactivity [25], buriability [26], composition [18], polarity [18], volume [18], charge transfer and donor capability [27], hydrogen-bond donor capability [28], secondary structure (i.e., alpha helix, beta structure, 
and coil) [29]. Initially, all 20 features are used. Feature selection is then applied to determine the most suitable combination of features that yields optimal performance.

For each residue $\alpha_{i}$ at position $i$, a peptide of running window size $w$ is represented by a vector of size equal to the number of features $(n)$ used: $\left[a v g_{\text {scale_- }}, a v g_{\text {scale }} 2, \ldots\right.$, $\left.a v g_{\text {scale } n}\right] . w$ in this study ranges from 5 to 29 in steps of 2 .

The average for each propensity scale is calculated by the following formula, where $i$ is the position index of a residue in the running peptide window, $c$ is the central residue position index in the peptide window, $|c-i|$ is the distance in residue number between residue $i$ and the central residue, $f$ is the linear weighting factor (in this study, $f=0.00,0.02,0.04,0.06,0.08,0.10), s_{i}$ is the propensity scale value of the residue at position $i$. For the property of PSSM, $s_{i}$ is the sum of the 20 PSSM log-likelihood values of residue $i$.

$$
\operatorname{avg}_{\text {scale }}=\frac{\sum_{i}(1-f|c-i|) s_{i}}{w}
$$

\section{Training and classification}

SVM is a machine learning algorithm proposed by Vapnik based on structural risk minimization principle of statistics learning theory [30]. It can be used to solve classification and regression problems. As determining whether a residue in a protein sequence belongs to an epitope is a binary classification problem, SVM would be useful for our purpose. In this study, LIBSVM, a well-known and powerful SVM package developed by Chang and Lin, is used to decipher epitope residues from non-epitope residues [31]. In the process of model development, we use radial basis function (RBF) as the kernel function in SVM. The formulation of RBF is defined in the following equation, where $x_{i}$ and $x_{j}$ are two data vectors, and $\gamma$ is a training parameter.

$$
R B F\left(x_{i}, x_{j}\right)=\exp \left(-\gamma\left\|x_{i}-x_{j}\right\|^{2}\right)
$$

Each running peptide window is labeled as either +1 if the central residue of the window belongs to an epitope or -1 if the central residue is not part of any epitope. The profile generated either by single or hybrid propensity scale method is first scaled to the range of $[-1,1]$ using built-in scaling program of LIBSVM.

For single propensity scale method, five-fold cross-validation is applied to the Sollner dataset with the following parameters: $-w_{+1}=20,-w_{-1}=1,-c=$ default, $-g=$ default. The procedure is iterated five times.

For hybrid propensity scale method, we use a more stringent three-way data split procedure to train and evaluate performance of BEEPro. We first divide the Sollner dataset into five distinct, non-overlapping sets: three for training (classifier learning), one for validation (parameter optimization and feature selection), and one for testing (performance evaluation). The procedure is iterated five times. The steps of model optimization include (in this order): (1) selection of optimal running window size, (2) optimization of $-c,-g$, (3) optimization of $-w_{+1}$, (4) feature selection, (5) optimization of linear weighting factor $f$, (6) re-optimization of $-w_{+1}$.

To further evaluate the performance of BEEPro, we perform five-fold cross-validation on each of the other datasets using exactly the same optimized setting obtained during training and validation with the Sollner dataset.

\section{Performance evaluation}

The area under the receiver operating curve (AUC) is used to assess performance during parameter selection. AUC is one of the most appropriate measures of performance as it is non-parametric and threshold independent. It is also independent of the number of positive and negative test cases. In addition, AUC is the recommended metric at a workshop organized by the National Institute of Allergy and Infectious Disease in 2006 [32]. AUC ranges from 0.5 to 1 , with 0.5 being random predictor, and 1 being perfect predictor.

Other metrics are also computed to allow more comprehensive comparison against previous studies. Sensitivity (SEN) and specificity (SPE) measure how well the classifier detects epitopes as epitopes and non-epitopes as nonepitopes, respectively. Matthew's correlation coefficient (MCC) takes under-prediction and over-prediction into consideration and is useful even when positive and negative test cases differ in size. Accuracy (ACC) is the proportion of correctly predicted residue. Positive predictive value (PPV) is the percentage of detected epitope residues that truly belong to an epitope. The following equations define these statistics, where TP, TN, FP, and FN denote the number of true positives, true negatives, false positives, and false negatives, respectively.

$$
\begin{aligned}
& S E N=\frac{T P}{T P+F N} \\
& S P E=\frac{T N}{T N+F P} \\
& M C C=\frac{T P \times T N-F P \times F N}{\sqrt{(T P+F P)(T P+F N)(T N+F P)(T N+F N)}} \\
& A C C=\frac{T P+T N}{T P+T N+F P+F N} \\
& P P V=\frac{T P}{T P+F P}
\end{aligned}
$$




\section{Results}

Prediction based on single propensity scale or position specific scoring matrix

In general, the prediction performance of single propensity scale methods improves as the size of window increases, with the exception of the accessible surface area scale, which decreases as window size increases, and the polarity scale, which fluctuates across different window sizes (Figure 1, Additional File 8: Supplementary Table 1). Among the four physicochemical propensity scales, Parker's hydrophilicity (AUC $=0.5855$ at $w=19$ ) and Karplus' flexibility (AUC $=0.5859$ ) scales have insignificant difference in performance, and both outperform Grantham's polarity (AUC $=0.5442$ ) and Janin's accessible surface area scale (AUC $=0.4863$ ). Accessible surface area has the worst performance of the four scales, with AUC value roughly being 0.49 . The amino acid ratio propensity scale (AUC $=0.6090$ ) outperforms the four physicochemical scales regardless of window size, and this gives us confidence to use this scale for the later hybrid model. PSSM outperforms amino acid ratio propensity scale and each of the four physicochemical properties, with AUC of 0.6786 at $w=19$.

\section{Hybrid propensity scale method significantly improves prediction performance}

There is a significant improvement when PSSM is incorporated into the hybrid propensity scale model and a slight improvement when amino acid ratio propensity scale is incorporated (Figure 2, Additional File 8: Supplementary Table 2). We also see here that the hybrid propensity scale method (AUC $=0.7049$ ) offers significant improvement over the single scale methods (AUC $=6786$ for PSSM). As with single propensity scale, the performance of the model improves as $w$ increases. We choose $w=19$ for further development as this is consistent with most literatures to date and would therefore allow more direct comparison between methods.

Feature selection allows us to determine the optimal parameter combination: $-w_{+1}=15,-w_{-1}=1,-c=1,-g=$ 10 , linear weighting factor $=0.08$. Finally, several features should be removed from feature list, including: Janin's accessible surface area, Grantham polarity, Bastolla's interactivity, and Zhou's buriability (Additional File: Supplementary Table 3). After parameter selection with the three-way data split approach, we arrived at the final performance $\mathrm{AUC}=0.9987, \mathrm{ACC}=0.9929, \mathrm{SEN}=0.9604$, $\mathrm{SPE}=0.9946, \mathrm{MCC}=0.9281, \mathrm{PPV}=0.9042$ for the Sollner dataset (Table 2).

\section{Performance comparison with existing methods}

Applying exactly the same feature extraction method and the same parameter setting as the optimized BEEPro model, we perform five-fold cross-validation on the independent datasets (Table 2).

For the linear epitope datasets AntiJen \#1 and \#2, HIV, Pellequer, PC, the AUC is 0.9930, 0.9907, 0.9907, 0.9874, and 0.9950 , respectively. The accuracy is $0.9731,0.9580$, $0.9454,0.9373$, and 0.9550 , respectively. BEEPro outperforms many other current linear epitope prediction methods, including LEPS [9], BepiPred [7], ABCPred [33], BCPred [15], and FBCPred [34] (Table 2).

Even for the conformational epitope dataset (Benchmark dataset), we are able to obtain AUC $=0.91, \mathrm{ACC}=0.92$, $\mathrm{SEN}=0.71, \mathrm{SPE}=0.94, \mathrm{MCC}=0.57, \mathrm{PPV}=0.52 . \mathrm{BEEPro}$ outperforms other conformational epitope predictors such as DiscoTope [35] and CEP [36] (Table 2). Although PPV value is not as high as for linear epitope datasets, it is still significantly higher than other current models. However, the sensitivity of BEEPro (0.71) for this Benchmark dataset

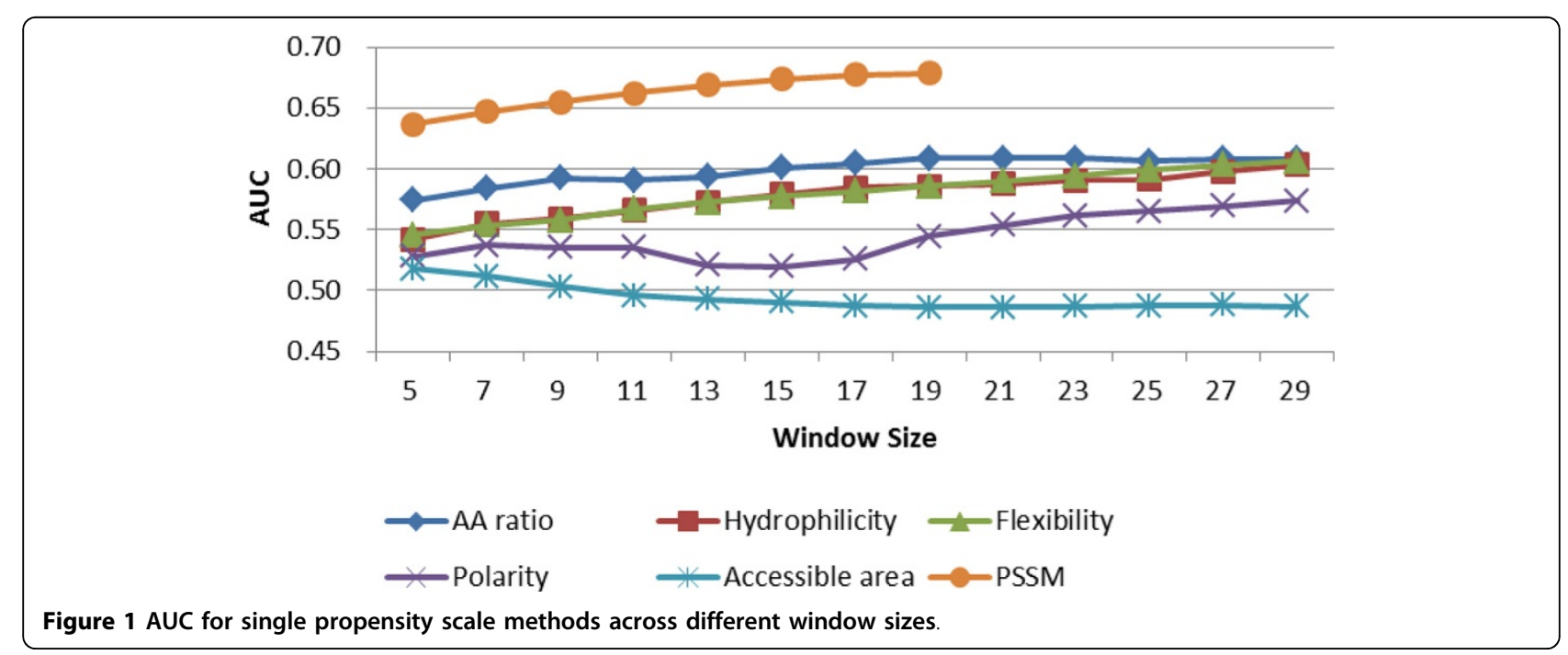




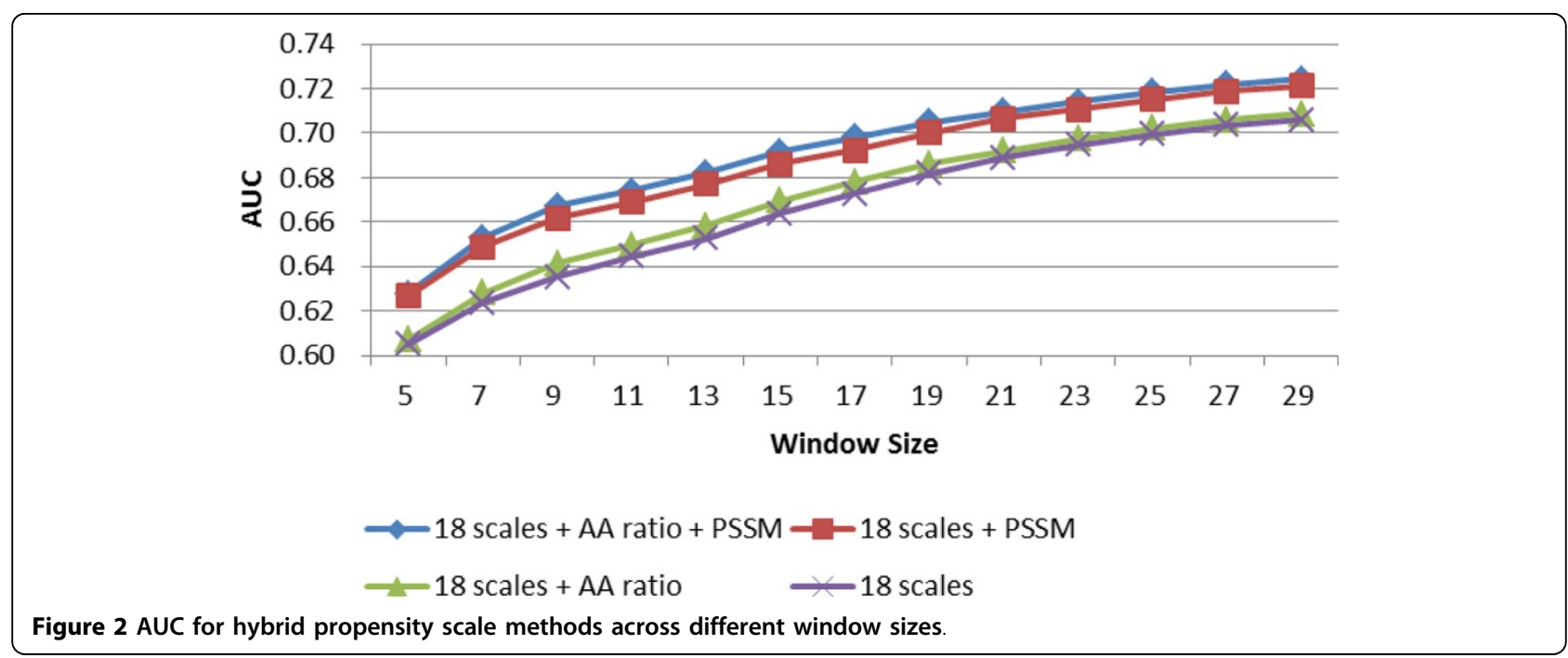

Table 2 Performance comparison of different methods using five-fold cross-validation for different datasets.

\begin{tabular}{|c|c|c|c|c|c|c|c|}
\hline Dataset & Method & AUC & ACC & SEN & SPE & $M C C$ & PPV \\
\hline Sollner & BEEPro & 0.9987 & 0.9929 & 0.9604 & 0.9946 & 0.9281 & 0.9042 \\
\hline AntiJen\#1 & BEEPro & 0.9930 & 0.9731 & 0.9680 & 0.9735 & 0.8491 & 0.7688 \\
\hline \multirow[t]{6}{*}{ AntiJen\#2 } & BEEPro & 0.9907 & 0.9580 & 0.9700 & 0.9562 & 0.8402 & 0.7668 \\
\hline & LEPS & NA & 0.7381 & 0.2672 & 0.8448 & 0.1010 & 0.2885 \\
\hline & BepiPred & NA & 0.5552 & 0.5179 & 0.5761 & 0.0604 & 0.2202 \\
\hline & ABCPred $_{0.8}$ & NA & 0.4470 & 0.6733 & 0.4040 & 0.0546 & 0.2183 \\
\hline & BCPred & NA & 0.5392 & 0.5884 & 0.5487 & 0.0893 & 0.2334 \\
\hline & FBCPred & NA & 0.5145 & 0.6031 & 0.5121 & 0.0673 & 0.2233 \\
\hline \multirow[t]{6}{*}{ HIV } & BEEPro & 0.9907 & 0.9454 & 0.9490 & 0.9433 & 0.8853 & 0.9098 \\
\hline & LEPS & NA & 0.6345 & 0.4833 & 0.7484 & 0.2276 & 0.7144 \\
\hline & BepiPred & 0.6000 & 0.5672 & 0.5016 & 0.6085 & 0.0972 & 0.6122 \\
\hline & ABCPred $_{0.7}$ & NA & 0.5659 & 0.8797 & 0.1465 & 0.0564 & 0.5633 \\
\hline & BCPred & NA & 0.6657 & 0.8018 & 0.5457 & 0.2980 & 0.6555 \\
\hline & FBCPred & NA & 0.6713 & 0.7320 & 0.5820 & 0.2781 & 0.6556 \\
\hline \multirow[t]{2}{*}{ Pellequer } & BEEPro & 0.9874 & 0.9373 & 0.9256 & 0.9435 & 0.8621 & 0.8935 \\
\hline & BepiPred & 0.6710 & NA & NA & NA & NA & NA \\
\hline \multirow[t]{6}{*}{$\overline{P C}$} & BEEPro & 0.9950 & 0.9550 & 0.9708 & 0.9468 & 0.9036 & 0.9058 \\
\hline & LEPS & NA & 0.6166 & 0.1278 & 0.8833 & 0.0365 & 0.4512 \\
\hline & BepiPred & NA & 0.5533 & 0.4823 & 0.5972 & 0.0749 & 0.3819 \\
\hline & ABCPred $_{0.8}$ & NA & 0.4889 & 0.6546 & 0.4026 & 0.0513 & 0.3621 \\
\hline & BCPred & NA & 0.5283 & 0.5092 & 0.5935 & 0.0443 & 0.3607 \\
\hline & FBCPred & NA & 0.5220 & 0.5103 & 0.5255 & 0.0317 & 0.3526 \\
\hline \multirow[t]{8}{*}{ Benchmark } & BEEPro & 0.9100 & 0.9200 & 0.7100 & 0.9400 & 0.5700 & 0.5200 \\
\hline & CBTOPE & 0.8900 & 0.8400 & 0.8000 & 0.8500 & NA & 0.3100 \\
\hline & DiscoTope & 0.6000 & 0.7500 & 0.4200 & 0.7900 & NA & 0.1600 \\
\hline & CEP & 0.5400 & 0.7400 & 0.3100 & 0.7800 & NA & 0.1100 \\
\hline & ClusPro(DOT) best model & 0.6900 & 0.8900 & 0.4500 & 0.9300 & NA & 0.3900 \\
\hline & Patch Dock best model & 0.6600 & 0.8500 & 0.4300 & 0.8900 & NA & 0.2600 \\
\hline & PSI-PRED best patch & 0.6000 & 0.8200 & 0.3300 & 0.8600 & NA & 0.1900 \\
\hline & ProMate & 0.5100 & 0.8400 & 0.0900 & 0.9200 & NA & 0.1000 \\
\hline
\end{tabular}

${ }^{1}$ LEPS, BepiPred, ABCPred, BCPred, FBCPred performances were previously compiled by Wang et al. [9]

${ }^{2}$ CBTOPE, DiscoTope, CEP, ClusPro, Patch Dock, PSI-PRED, ProMate performances were previously compiled by Ansari and Raghava [13] 
is not as high as CBTOPE (0.80), and this could be explained by BEEPro's significantly higher specificity.

\section{Discussion \\ Hybrid method performs better than single propensity scale method}

Blythe and Flower determined in 2005 that the best single propensity scale performs only marginally better than random prediction [4]. Several studies have later demonstrated that the use of a combination of propensity scales for feature representation could have better results than using single propensity scale [5]. Our results agree with this statement, as we see a significant improvement in performance between single propensity scale methods and hybrid models. This reflects the complex nature of epitopes, as more than one property is needed to reflect the epitope peptide profile.

\section{Evolutionary information is effective for B-cell epitope identification}

Evolutionary information, encoded as PSSM generated by PSI-BLAST, has been used to improve prediction performance of other biological prediction problems such as RNA binding sites and protein cellular localization [37-40]. In the field of B-cell epitope prediction, El-Manzalawy et al. have shown that PSSM outperforms selected single physicochemical scales, a result similarly observed in this study [3]. We extend this observation and demonstrate in this paper that while PSSM alone may be insufficient, combining PSSM with other propensity scales does improve the hybrid propensity scale model markedly, implicating the significance of evolutionary information and sequence conservation as an important determining factor for a peptide's immunogenic property.

\section{Effects of physicochemical propensity scales}

We have constructed a prediction method for B-cell epitopes using support vector machine. The finalized feature list is based on amino acid ratio propensity scale, PSSM, and 14 physicochemical propensity scales that reflect properties of antigenicity, hydrophilicity, flexibility, composition, volume, charge transfer and donor capability, hydrogen bond donor capability, and secondary structure such as alpha helical structure, beta structure, and coil structure. We have included chemical properties such as charge transfer and donor, and hydrogen bond donor scales in the development of our model, which have not been attempted in previous literatures to the best of our knowledge. It is possible that these chemical properties could be relevant in determining the chemical behaviors between antigens and antibodies. We have also applied for the first time Bastolla's interactivity scale [25] and Zhou's buriability scale [26], but these two scales fail to improve our hybrid propensity scale model.

\section{Generality of BEEPro on different datasets}

Sollner et al. curated their linear B-cell epitope dataset from the IEDB database using a series of filtering steps that allow correlation of annotated epitopes with functional relevance. This Sollner dataset is used for training and optimization of BEEPro in our study. Although we have trained our model using a more stringent protective linear B-cell epitope dataset, we have also shown that the same feature representation method and parameter setting can be extended to general linear B-cell epitopes and even to conformational B-cell epitopes with high performance. Compared with other current prediction systems of linear and conformational epitopes, our method has a superior performance in area under curve, accuracy, Matthew's correlation coefficient, positive predictive value, sensitivity, and specificity.

The observation that BEEPro does not perform as well in the Benchmark dataset, which consists of conformational epitopes, is not surprising as the model was originally optimized using a linear epitope dataset. However, BEEPro still outperforms current structurebased prediction methods in classifying conformational epitope positions, and this would echo previous report that sequence-based predictor can work as a complement to current structure-based prediction methods [13]. BEEPro can also be applied when structural information of antibody-antigen complex is not available.

It is promising to note that PPV of BEEPro is about 0.90 0.91 for linear epitopes, and 0.52 for conformational epitopes, as this could imply a more cost-effective approach to screen for possible peptide vaccine candidates. Also, BEEPro has been shown to excel in independent datasets of varying epitope density (from $5.04 \%$ to $37.55 \%$ ), and this would reflect applications in real world where epitopes take up only a small portion of an antigen sequence.

\section{Conclusions}

In this paper, we describe BEEPro, a method to predict Bcell epitopes using evolutionary information, amino acid ratio propensity scale, and 14 physicochemical propensity scales, for a total of 16 features. The 14 physicochemical propensity scales in BEEPro reflect information on antigenicity, hydrophilicity, flexibility, composition, volume, charge transfer and donor capability, hydrogen bond donor capability, and secondary structure such as alpha helical structure, beta structure, and coil structure. The five-fold cross-validation accuracy on six linear B-cell epitope datasets ranges from $93.73 \%$ to $99.29 \%$, with AUC ranging from 0.9874 to 0.9987 . In addition, the five-fold cross-validation accuracy on one benchmark conformational B-cell epitope dataset is $92.14 \%$, with AUC of 0.9066. We have shown that BEEPro outperformed other sequence-based and structure-based prediction methods. 


\section{Additional material}

Additional file 1: The Sollner dataset.
Additional file 2: The AntiJen\#1 dataset.
Additional file 3: The AntiJen\#2 dataset.
Additional file 4: The HIV dataset.
Additional file 5: The Pellequer dataset.
Additional file 6: The PC dataset.
Additional file 7: The Benchmark dataset.
Additional file 8: Supplementary tables on optimization of hybrid
propensity scale method.

\section{Authors' contributions}

SYHL designed and implemented the system, analyzed the data, and drafted the manuscript. CWC provided computational assistance. ECYS supervised the project and revised the manuscript. All the authors read and approved the final manuscript.

\section{Competing interests}

The authors declare that they have no competing interests.

\section{Acknowledgements}

The research was supported in part by National Science Council under grant NSC100-2221-E-038-012 and NSC101-2221-E-038-014 to Emily Chia-Yu Su. Scott Yi-Heng Lin would like to thank the National Science Council for providing undergraduate student research scholarship NSC100-2815-C-038004-E and NSC101-2815-C-038-005-E.

\section{Declarations}

The publication costs for this article were funded by National Science Council under grant NSC101-2221-E-038-014.

This article has been published as part of BMC Bioinformatics Volume 14 Supplement 2, 2013: Selected articles from the Eleventh Asia Pacific Bioinformatics Conference (APBC 2013): Bioinformatics. The full contents of the supplement are available online at http://www.biomedcentral.com/ bmcbioinformatics/supplements/14/S2.

\section{Author details}

${ }^{1}$ School of Medicine, College of Medicine, Taipei Medical University, Taipei, Taiwan. ${ }^{2}$ Institute of Information Sciences, Academia Sinica, Taipei, Taiwan. ${ }^{3}$ Graduate Institute of Biomedical Informatics, Taipei Medical University, Taipei, Taiwan.

Published: 21 January 2013

\section{References}

1. EL-Manzalawy $Y$, Honavar V: Recent advances in B-cell epitope prediction methods. Immunome Res 2010, 6(Suppl2):S2.

2. Sollner J, Grohmann R, Rapberger R, Perco P, Lukas A, Mayer B: Analysis and prediction of protective continuous $B$-cell epitopes on pathogen proteins. Immunome Research 2008, 4(1):1.

3. EL-Manzalawy Y, Dobbs D, Honavar V: Predicting Protective Linear B-Cell Epitopes Using Evolutionary Information. IEEE International Conference on Bioinformatics and Biomedicine 2008, 289-292.

4. Blythe $M$, Flower D: Benchmarking B cell epitope prediction underperformance of existing methods. Protein Sci 2005, 14:246-248.

5. Rubinstein N, Mayrose I, Pupko T: A machine-learning approach for predicting B-cell epitopes. Mol Immunol 2009, 46:840-847.

6. Chen J, Liu H, Yang J, Chou KC: Prediction of linear B-cell epitopes using amino acid pair antigenicity scale. Amino Acids 2007, 33(3):423-428.

7. Larsen J, Lund $O$, Nielsen M: Improved method for predicting linear B-cell epitopes. Immunome Res 2006, 2:2.

8. Toseland C, Clayton D, McSparron H, Hemsley S, Blythe M, Paine K, Doytchinova I, Guan P, Hattotuwagama C, Flower D: AntiJen: a quantitative immunology database integrating functional, thermodynamic, kinetic, biophysical, and cellular data. Immunome Res 2005, 1(1):4.

9. Wang H-W, Lin Y-C, Pai T-W, Chang H-T: Prediction of B-cell Linear Epitopes with a Combination of Support Vector Machine Classification and Amino Acid Propensity Identification. J Biomed Biotechnol 2011, 2011.

10. Korber B, Brander C, Haynes B, Koup R, Moore J, Walker B, Watkins D: HIV Immunology and HIV/SIV Vaccine Databases 2003 Los Alamos, New Mexico: Los Alamos National Laboratory, Theoretical Biology and Biophysics; 2003.

11. Pellequer J, Westhof E, Van Regenmortel M: Correlation between the location of antigenic sites and the prediction of turns in proteins. Immunol Lett 1993, 36:83-99.

12. Ponomarenko J, Bourne P: Antibody-protein interactions: benchmark datasets and prediction tools evaluation. BMC Struct Biol 2007, 7(1):64.

13. Ansari H, Raghava G: Identification of conformational B-cell Epitopes in an antigen from its primary sequence. Immunome Res 2010, 6(1):6.

14. Li W, Godzik A: Cd-hit: a fast program for clustering and comparing large sets of protein or nucleotide sequences. Bioinformatics 2006, 22(13):1658-1659.

15. El-Manzalawy Y, Dobbs D, Honavar V: Predicting linear B-cell epitopes using string kernels. J of Mol Recognit 2008, 21(4):243-255.

16. Parker J, Guo D, Hodges R: New Hydrophilicity Scale Derived from HighPerformance Liquid Chromatography Peptide Retention Data: Correlation of Predicted Surface Residues with Antigenicity and X-rayderived Accessible Sites. Biochemistry 1986, 25:5425-5432.

17. Karplus P, Schulz G: Prediction of chain flexibility in proteins: a tool for the selection of peptide antigen. Naturwissenschaften 1985, 72:212-213.

18. Grantham R: Amino acid difference formula to help explain protein evolution. Science 1974, 185(862-864).

19. Janin J, Wodak S: Conformation of amino acid side-chains in proteins. J Mol Biol 1978, 125:357-386

20. Kawashima S, Pokarowski P, Pokarowska M, Kolinski A, Katayama T, Kanehisa M: AAindex: amino acid index database, progress report 2008. Nucleic Acids Research 2008, 36(suppl 1):D202-D205.

21. Kolaskar A, Tongaonkar P: A semi-empirical method for prediction of antigenic determinants on protein antigens. FEBS Lett 1990, 276:172-174

22. Hopp T, Woods K: Prediction of protein antigenic determinants from amino acid sequences. Proc Natl Acad Sci 1981, 78:3824-3828.

23. Ponnuswamy $P$, Prabhakaran M, Manavalan P: Hydrophobic packing and spatial arrangement of amino acid residues in globular proteins. Biochim Biophys Acta 1980, 623:301-326.

24. Bhaskaran $R$, Ponnuswamy P: Positional flexibilities of amino acid residues in globular proteins. Int J Peptide Protein Res 1988, 32:241-255.

25. Bastolla U, Porto M, Roman HE, Vendruscolo M: Principal eigenvector of contact matrices and hydrophobicity profiles in proteins. Proteins: Struct, Funct, Bioinf 2005, 58(1):22-30.

26. Zhou $H$, Zhou $Y$ : Quantifying the effect of burial of amino acid residues on protein stability. Proteins: Struct, Funct, Bioinf 2004, 54:315-322.

27. Charton M, Charton BI: The dependence of the Chou-Fasman parameters on amino acid side chain structure. J Theor Biol 1983, 102(1):121-134.

28. Fauchere J, Charton M, Kier L, Verloop A, Pliska V: Amino acid side chain parameters for correlation studies in biology and pharmacology. Int $\mathrm{J}$ Pept Protein Res 1988, 32(4):269-278.

29. Nagano K: Logical analysis of the mechanism of protein folding: I. predictions of helices, loops and beta-structures from primary structure. J Mol Biol 1973, 75(2):401-420.

30. Vapnik V: The nature of statistical learning theory Springer; 1995

31. Chang C-C, Lin C-J: LIBSVM: A library for support vector machines. ACM Trans Intell Syst Technol 2011, 2(3):1-27.

32. Greenbaum JA, Andersen PH, Blythe MJ, Bui H-H, Cachau RE, Crowe J, Davies M, Kolaskar AS, Lund O, Morrison S, et al: Towards a consensus on datasets and evaluation metrics for developing B-cell epitope prediction tools. Jounral of Molecular Recognition 2007, 20:75-82.

33. Saha S, Raghava GPS: Prediction of continuous B-cell epitopes in an antigen using recurrent neural network. Proteins: Structure, Function, and Bioinformatics 2006, 65(1):40-48.

34. EL-Manzalawy $Y$, Dobbs D, Honavar V: Predicting flexible length linear Bcell epitopes. Comput Syst Bioinformatics Conf 2008, 7:121-132.

35. Haste Andersen P, Nielsen M, Lund O: Prediction of residues in discontinuous B-cell epitopes using protein 3D structures. Protein Science 2006, 15(11):2558-2567. 
36. Kulkarni-Kale U, Bhosle S, Kolaskar AS: CEP: a conformational epitope prediction server. Nucleic Acids Research 33(suppl 2):W168-W171.

37. Cheng C-W, Su EC-Y, Hwang J-K, Sung T-Y, Hsu W-L: Predicting RNAbinding sites of proteins using support vector machines and evolutionary information. BMC Bioinformatics 2008, 9:S6.

38. Pu X, Guo J, Leung $H$, Lin Y: Prediction of membrane proteins types from sequences and position-specific scoring matrices. Journal of Theoretical Biology 2007, 247:259-265.

39. Wang $Y$, Xue $Z$, Shen $G, X u$ J: PRINTR: Prediction of RNA binding sites in proteins using SVM and profiles. Amino Acids 2008, 35:295-302.

40. Mundra P, Kumar M, Kumar KK, Jayaraman VK, Kulkarni BD: Using pseudo amino acid composition to predict protein subnuclear localization: approached with PSSM. Pattern Recognition Letters 2007, 28:1610-1615.

doi:10.1186/1471-2105-14-S2-S10

Cite this article as: Lin et al:: Prediction of B-cell epitopes using

evolutionary information and propensity scales. BMC Bioinformatics 2013

14(Suppl 2):S10.

\section{Submit your next manuscript to BioMed Central} and take full advantage of:

- Convenient online submission

- Thorough peer review

- No space constraints or color figure charges

- Immediate publication on acceptance

- Inclusion in PubMed, CAS, Scopus and Google Scholar

- Research which is freely available for redistribution

Submit your manuscript at www.biomedcentral.com/submit
Ciomed Central 